\title{
Detection of exacerbations in asthma based on electronic diary data: results from the 1-year prospective BIOAIR study
}

\author{
Maciej Kupczyk, ${ }^{1}$ Shushila Haque, ${ }^{1}$ Peter J Sterk, ${ }^{2}$ Ewa Niżankowska-Mogilnicka, ${ }^{3}$ \\ Alberto Papi, ${ }^{4}$ Elisabeth $\mathrm{H} \mathrm{Bel}_{1}{ }^{2}$ Pascal Chanez, ${ }^{5}$ Barbro Dahlén, ${ }^{1}$ Mina Gaga, ${ }^{6}$ \\ Mark Gjomarkaj, ${ }_{1}^{7}$ Peter H Howarth, ${ }^{8}$ Sebastian L Johnston, ${ }_{1}^{9}$ Guy F Joos, ${ }^{10}$ \\ Frank Kanniess, ${ }^{11}$ Eleni Tzortzaki, ${ }^{12}$ Anna James, ${ }^{1}$ Roelinde J M Middelveld, ${ }^{1}$ \\ Sven-Erik Dahlén, ${ }^{1}$ on behalf of the BIOAIR investigators
}

- Additional material is published online only. To view please visit the journal online (http://dx.doi.org/10.1136/ thoraxjn-2012-201815).

For numbered affiliations see end of article.

\section{Correspondence to} Dr Maciej Kupczyk, Centre for Allergy Research, Karolinska Institutet, IMM Physiology, PO Box 287, Stockholm SE-171 77, Sweden; maciek.kupczyk@ki.se

Received 25 February 2012 Revised 13 February 2013 Accepted 17 March 2013

Published Online First 6 April 2013
To cite: Kupczyk $M$, Haque S, Sterk PJ, et al. Thorax 2013;68:611-618.

\section{ABSTRACT \\ Background Objective measures are required that may be used as a proxy for exacerbations in asthma. The aim was to determine the sensitivity and specificity of electronic diary data to detect severe exacerbations (SEs) of asthma. A secondary aim was to identify phenotypic variables associated with a higher risk of exacerbation. \\ Methods In the BIOAIR study, 169 patients with asthma (93 severe $(\mathrm{SA}) ; 76$ mild to moderate $(\mathrm{MA})$ ) recorded lung function, symptoms and medication use in electronic diaries for 1 year. Data were analysed using receiver-operator characteristics curves and related to physician-diagnosed exacerbations. Medical history and baseline clinical data were used to assess risk of exacerbation.}

Results of 122 physician-diagnosed exacerbations, 104 occurred in the SA group (1.1 per patient/year), 18 in the MA group (0.2 per patient/year) and 63 were severe using American Thoracic Society/European Respiratory Society criteria. During exacerbations, peak expiratory flow (PEF) and forced expiratory volume in $1 \mathrm{~s}$ significantly decreased, whereas day and night symptoms significantly increased. An algorithm combining a 20\% decrease in PEF or a $20 \%$ increase in day symptoms on 2 consecutive days was able to detect SEs with $65 \%$ sensitivity and $95 \%$ specificity. The strongest risk factors for SEs were low Asthma Control Questionnaire score, sputum eosinophils $\geq 3 \%$, body mass index $>25$ and low quality of life (St George's Respiratory

Questionnaire), with ORs between 3.61 and 2.22 $(p<0.05)$.

Conclusions Regular electronic monitoring of PEF and asthma symptoms provides an acceptable sensitivity and specificity for the detection of SEs and may be suitable for personal internet-based monitoring of asthma control.

\section{INTRODUCTION}

Asthma exacerbations involve episodes of acute worsening of the disease. The increase in symptoms (shortness of breath, cough, wheezing and chest tightness) results in a limitation of daily activities and the need for unscheduled healthcare intervention. ${ }^{1}{ }^{2}$ Exacerbations may also involve severe, life-threatening events, and they represent a great burden for the patients and for the healthcare systems. The level of asthma control is defined by

\section{Key messages}

What is the key question?

- Which variables (symptoms, rescue medication use or lung function) represent the highest sensitivity and specificity to detect severe exacerbations in asthma patients?

What is the bottom line?

- Monitoring of PEF and symptoms of asthma gives the highest sensitivity and specificity for use as a proxy of severe exacerbations in asthma and may be used in personal, electronic or internet-based monitoring of asthma control.

Why to read on?

- The study identifies and validates objective criteria for the capturing of severe exacerbations.

clinical symptoms, and the treatment necessary to maintain this control and to avoid exacerbations. ${ }^{134}$ Accordingly, definitions of severe, problematic or difficult-to-treat asthma incorporate exacerbations as a component to assess severity, in adults ${ }^{56}$ and in children. ${ }^{7}$ When testing the efficacy of new drug treatments in asthma, exacerbation frequency is often used as a primary outcome variable. ${ }^{8}$ To identify exacerbations in everyday medical practise and in clinical trials, a wide range of unvalidated criteria have been applied. ${ }^{8}$ Recent American Thoracic Society/European Respiratory Society (ATS/ERS) guidelines ${ }^{8}$ defined severe exacerbations (SEs) according to the need for systemic corticosteroid use, a visit to an emergency department or hospital admission, whereas moderate exacerbations were defined by a deterioration in symptoms, decrease in lung function and increase in rescue medication use. However, the guidelines recognised that there is a remarkable absence of studies that have analysed and validated objective outcomes which may be used in the future to define exacerbations in asthma and thus can be regarded as a proxy for exacerbation. 
The primary goal of our study was to analyse the sensitivity and specificity of different variables to detect SEs in patients with asthma based on data collected during a 1-year follow-up with electronic diaries in the BIOAIR (Longitudinal Assessment of Clinical Course and Biomarkers in Severe Chronic Airway Disease) cohort of comprehensively examined patients. The electronic diary data included lung function (peak expiratory flow (PEF) and forced expiratory volume in $1 \mathrm{~s}\left(\mathrm{FEV}_{1}\right)$ ), use of a short-acting $\beta$ agonist (SABA) as rescue medication, day and night symptoms, and limitation of daily activities. The analysis was performed to ascertain whether or not there were patterns in the electronic diary recordings that related to independently determined physician-diagnosed SEs. The overall aim of this analysis was to define the thresholds of various symptoms and lung function measurements that distinguish severe from moderate exacerbations at the time that they occur, as such values may be necessary for the future development of internet-aided personalised healthcare plans ${ }^{9}$ or in clinical trials. The secondary goal of our study was to analyse potential risk factors for the development of SEs from medical history and baseline clinical data.

\section{METHODS}

\section{Study design}

The BIOAIR trial was a project on biomarkers and clinical outcomes partly supported by the European Union with a particular focus on severe asthma. Patients with asthma were screened and allocated to severe (SA) or mild to moderate asthma (MA) groups. After a 4-week period of treatment optimisation, patients were randomised to a systemic steroid versus placebo crossover intervention during the biomarker characterisation phase following which they were followed up for at least 1 year (figure 1). Information regarding lung function, biomarkers (induced sputum, peripheral blood, exhaled nitric oxide), atopy, medical history, asthma control (evaluated with the use of the Juniper Asthma Control Questionnaire (ACQ) $)^{10}$ and quality of life (evaluated with the use of St Georges Respiratory Questionnaire (SGRQ)) ${ }^{11}$ was collected at baseline. In addition, lung function (PEF and $\mathrm{FEV}_{1}$ ), rescue medication use (SABA), day and night symptoms, and limitation of activities were recorded daily using electronic diaries (Vitalograph Electronic $\mathrm{PEF} / \mathrm{FEV}_{1}$ Diary, XM version, Vitalograph Ltd, Buckingham, UK). For further details regarding the exact questions, scales used and definitions of asthma severity please refer to the online repository materials. The study was registered at clinicaltrials. gov (NCT00555607) and the protocol was approved by the local ethics committees and drug regulatory authorities in the 12 participating centres. ${ }^{12}$

\section{Exacerbations}

ATS/ERS recommendations ${ }^{8}$ were used to define exacerbations as events clinically identified to be outside the patient's usual range of day-to-day asthma variation, and requiring a change in controller treatment. A SE was retrospectively defined as a worsening requiring at least 3 days' use of oral corticosteroids, or as an increase in systemic corticosteroids from an individual maintenance dose, or as a visit to the emergency room or hospitalisation. Likewise, a moderate exacerbation was defined as a period requiring a change in treatment, but not fulfilling the criteria of a SE. In line with the recommendations of the ATS/ERS task force ${ }^{8}$ mild asthma exacerbations were not identified as they could not be distinguished from a transient loss of asthma control. During the study, the patients were supplied with written cards advising them to contact the study physician or nurse immediately (all centres available $24 \mathrm{~h} /$ day and 7 days/week) in case of a troublesome loss of asthma control (increase in symptoms and rescue medication use, decrease in daily activities or quality of life). Based on medical history, symptoms, medication use and additional tests, the responsible physician then labelled the event as an asthma exacerbation. Day 1 of an exacerbation was defined as the day on which the subject visited the clinic due to an exacerbation (figure 1). For further details please refer to the online repository.

\section{Data collection and statistical analysis}

Data were entered into a central database through a web-based Case Record Form system developed specifically for the BIOAIR study. Patient baseline characteristics are expressed as mean \pm SEM. Analysis of variance, followed by Fisher's protected least significant difference test, was used to test differences between the groups. The values collected during exacerbations were compared with the same subject's baseline values, defined as the average of the last 10 days of the treatment optimisation period (figure 1). These values were selected as a reasonable approximation of 'best values'. Other comparators used were 2, 5 and 7 days respectively before the start of an exacerbation. Values during exacerbations were analysed in three different ways: on the day of reported exacerbation (values measured when the patient visited the clinic due to symptoms of an exacerbation); the average of the values from the two worst consecutive days (peak drop in PEF) during the 10-day exacerbation window after the first visit; and the average of 10 days from the start of the exacerbation. The results

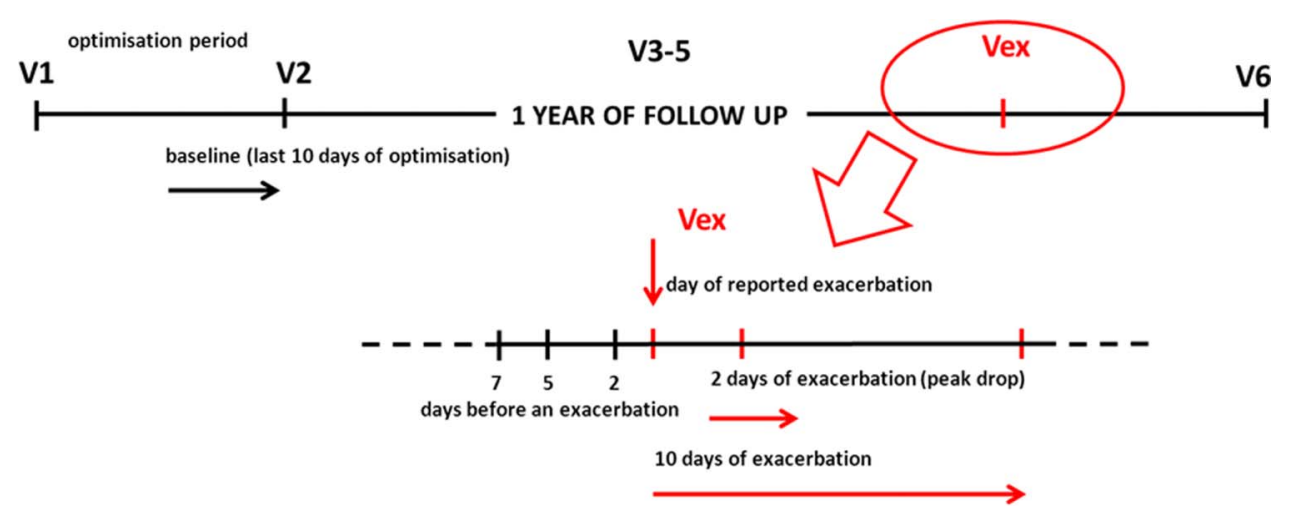

Figure 1 Study flow chart of the 1-year follow-up. Lower line represents illustration of how calculations were made during the exacerbation period requiring Vex. This figure is only reproduced in colour in the online version. 
are presented as percentage change from baseline. To evaluate the statistical significance of the findings, the Mann-Whitney test was used. To study the sensitivity and specificity of different parameters for the detection of exacerbations, receiver-operator characteristics (ROC) curves were analysed. ROC curves usually compare a certain variable measured in 'controls' and 'cases' to determine the cut-off values for this variable that give the optimal specificity and selectivity for defining a particular disease. Here 'cases' were represented by the change in a certain variable measured on the first 2 days of a doctor-diagnosed exacerbation compared with baseline. 'Controls' were represented by the change in a certain variable measured on all normal days outside of the exacerbation period compared with baseline, to provide an indication of normal fluctuations in the relevant variable. The variables studied were PEF, $\mathrm{FEV}_{1}$, day and night symptoms, and rescue medication use, and change in these values, either on the first 2 days of the exacerbation or on all days outside of the exacerbation period, were compared with baseline (patient's best as a mean of the last 10 days of the optimisation period).

To identify potential factors associated with the development of exacerbations, subjects with reported exacerbations were compared with those who did not have exacerbations during the 1-year follow-up period. A multivariate logistic regression model including all potential predictors with a univariate $p$ value $<0.1$ was built, and adjusted for atopy, sex, age and smoking status. SPSS V.17.0 software was used for statistical analysis. A $\mathrm{p}$ value $<0.05$ was considered a statistically significant difference for all tests.

\section{RESULTS}

\section{Patient characteristics}

A total of 169 subjects were screened and included in the BIOAIR study. ${ }^{12}$ Table 1 displays their demographic data and baseline characteristics. In comparison with MA, patients with SA were older (50 vs 42.2 years), had a higher body mass index
(BMI) $\left(28.5\right.$ vs $\left.25.0 \mathrm{~kg} / \mathrm{m}^{2}\right)$, lower lung function $\left(\mathrm{FEV}_{1} \%\right.$ of predicted: $70.4 \%$ vs $88.7 \%$ ) and poorer asthma control (Juniper ACQ score 2.03 vs 1.03 ). Comprehensive data on the patient cohorts are reported elsewhere. ${ }^{12}$ As many as 53 patients with SA (56.9\% of those screened and randomised) and 53 with MA (69.7\%) completed 1-year of follow-up. Mean adherence to the study protocol regarding daily measurements with the electronic diaries reached $86.6 \%$ in patients with SA and $79.4 \%$ in those with MA. Only events for which $>60 \%$ of days were completed in patient diaries have been used for further calculations.

\section{Number of exacerbations}

During the 1-year follow-up, 68 patients $(40.2 \%$ of the whole asthma cohort) had a total of 122 exacerbations in the range of one to six events per year. Figure 2A displays the distribution of exacerbations in the whole asthma cohort and figure $2 \mathrm{~B}$ the monthly distribution over the 1-year follow-up. The average number of exacerbations per patient per year was 0.72 for the whole asthma cohort.

The vast majority of exacerbations occurred in the SA group ( $n=93$ patients) in which a total of 104 exacerbations were recorded. The average number of exacerbations per patient per year was 1.12 in the SA group, although 41 of 93 patients in the SA group did not have any exacerbations. Out of patients who did have exacerbations, the true number of exacerbations per year was therefore $2.0(104 / 52)$. Forty-four exacerbations in the SA group (42.3\%) were severe but did not require hospitalisation, and in 14 cases (13.7\%) hospitalisation was necessary.

In contrast to SA, only 16 patients in the MA group (22.2\%) had a total of 18 exacerbations during the 1-year follow-up. The number of exacerbations per patient per year was 0.24 . Five exacerbations were severe $(27.8 \%)$. No hospitalisations due to asthma exacerbations were reported in the MA group.

Table 1 Demographic data and baseline characteristics of the study cohort (mean values $( \pm$ SEM), unless stated differently)

\begin{tabular}{|c|c|c|c|}
\hline & Severe asthma & Mild to moderate asthma & $p$ Value \\
\hline Patients in the BIOAIR cohort (n) & 93 & 76 & ND \\
\hline Age (years) (min-max) & $50.0 \pm 1.3(18-72)$ & $42.2 \pm 1.5(20-70)$ & $0.001 *$ \\
\hline Women (\%) & 58 & 61 & $0.982 \dagger$ \\
\hline $\mathrm{FEV}_{1}(\%$ pred $)$ & $70.4 \pm 2.1$ & $88.7 \pm 2.1$ & $<0.0001 *$ \\
\hline $\mathrm{FEV}_{1}$ (litres) & $2.04 \pm 0.08$ & $2.79 \pm 0.08$ & $<0.0001^{*}$ \\
\hline $\mathrm{FEV}_{1} / \mathrm{FVC}$ & $0.67 \pm 0.01$ & $0.70 \pm 0.01$ & $0.093^{*}$ \\
\hline Reversibility ( $\%$ of change) & $9.4 \pm 0.8$ & $10.6 \pm 0.7$ & $0.192^{*}$ \\
\hline ICS (median (mean \pm SD)) Beclomethasone eq. & $1600 \mu g^{*}(2064 \pm 939.7)$ & $800 \mu g(614 \pm 218.6)$ & $<0.0001^{*}$ \\
\hline OCS (median (mean $\pm S D ;$ min-max)) prednisolone eq. & $10 \mathrm{mg}(14.15 \pm 11.8 ; 2-50)$ & - & ND \\
\hline BMI $\left(\mathrm{kg} / \mathrm{m}^{2}\right)$ & $28.5 \pm 0.6$ & $25.0 \pm 0.4$ & $<0.0001^{*}$ \\
\hline ACQ (Juniper) & $2.03 \pm 0.1$ & $1.03 \pm 0.7$ & $<0.0001^{*}$ \\
\hline QoL (SGRQ) & $45.9 \pm 2.1$ & $22.5 \pm 2.0$ & $<0.0001^{*}$ \\
\hline CRP (mg/litre) & $6.1 \pm 0.9$ & $3.5 \pm 0.6$ & $0.092^{*}$ \\
\hline Atopy (\%) & 43 & 48 & $0.642 \dagger$ \\
\hline $\mathrm{F}_{\mathrm{E}} \mathrm{NO}(\mathrm{ppb})$ & $46.3 \pm 6.2$ & $40.1 \pm 4.1$ & $0.962^{*}$ \\
\hline Sputum cells $\left(\times 10^{6}\right)$ & $3.34 \pm 1.02 *$ & $1.83 \pm 0.34$ & $0.455^{*}$ \\
\hline Sputum eosinophils (\%) & $16.7 \pm 3.49^{*}$ & $5.79 \pm 1.71$ & $0.018^{*}$ \\
\hline Sputum neutrophils (\%) & $42.2 \pm 3.7$ & $44.2 \pm 4.4$ & $0.338^{*}$ \\
\hline \multicolumn{4}{|c|}{$\begin{array}{l}\text { Atopy defined as at least one positive skin prick test. } \mathbf{p}<0.05 \\
{ }^{*} \text { Mann-Whitney U test. } \\
+\chi^{2} \text { test. }^{12} \\
\text { ACQ, Asthma Control Questionnaire; BIOAIR, Longitudinal Assessment of Clinical Course and Biomarkers in Severe Chronic Airway Disease; BMI, body mass index; CRP, serum C-reactive } \\
\text { protein; FEV } 1 \text {, forced expiratory volume in } 1 \text { s; FVC, forced vital capacity; ICS, inhaled corticosteroids; ND, not determined; OCS, oral corticosteroid; QoL, quality of life; SGRQ, } \\
\text { St George's Respiratory Questionnaire. }\end{array}$} \\
\hline
\end{tabular}



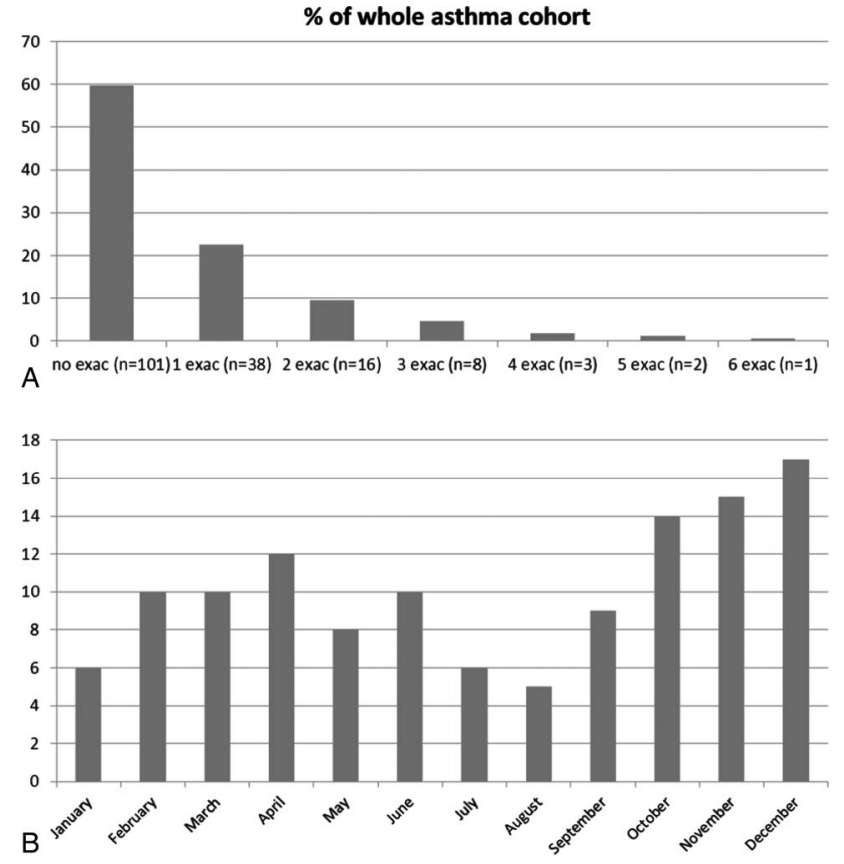

Figure 2 Percentage and number ( $\mathrm{n}$ ) of patients with asthma exacerbations (exac) during 1 year of follow-up in the whole asthma cohort $((A) n=169)$, and the monthly distribution of exacerbations over the 1-year period $(B)$.

In total, 63 exacerbations were classified as severe based on ATS/ERS criteria. ${ }^{8}$ In the current report, data regarding all SEs in both groups (severe and mild-to-moderate groups combined) are reported. Complete data on all exacerbations are displayed for the whole asthma group, and for SA and MA groups separately, in the online repository.

\section{Changes recorded during SEs}

Figure 3 displays changes in the studied variables during different periods in relation to the day of exacerbation. The data are expressed as a percentage of the personal best value recorded after treatment optimisation at the beginning of the study (figure 1 shows how calculations have been made). For all variables, there was a gradually increasing change from 7 days before an exacerbation until 2 days before.

The maximal drop in PEF (the average of the values from the two worst consecutive days vs baseline) was $19.8 \%(\mathrm{p}=0.0001)$. During the 10-day period following an exacerbation, a $12.2 \%$ decrease in PEF was observed $(\mathrm{p}=0.017)$ (figure 3A). During the 2 days of maximum deterioration, $\mathrm{FEV}_{1}$ decreased by $20.4 \%(\mathrm{p}=0.0007)$, and there was a 9.7\% decrease in $\mathrm{FEV}_{1}$ during the 10 days of an exacerbation $(\mathrm{p}=0.04)$ (figure $3 \mathrm{~B}$ ).

There were no significant changes in the use of SABAs before or during the exacerbations (figure $3 \mathrm{C}$ ), although the numerical value was 0.44 puffs higher on the day of the reported exacerbation.

With respect to day and night symptoms, there was a significant increase $(41.0 \%)$ in day symptoms on the day of the reported exacerbation compared with baseline $(\mathrm{p}=0.002)$ but no change in night symptoms $(\mathrm{p}=0.14$; figure $3 \mathrm{D}, \mathrm{E})$.

For the variable 'limitation of daily activities', a significant increase was observed $(63.1 \%, \mathrm{p}=0.029)$ when the whole 10 -day period of exacerbation was analysed.
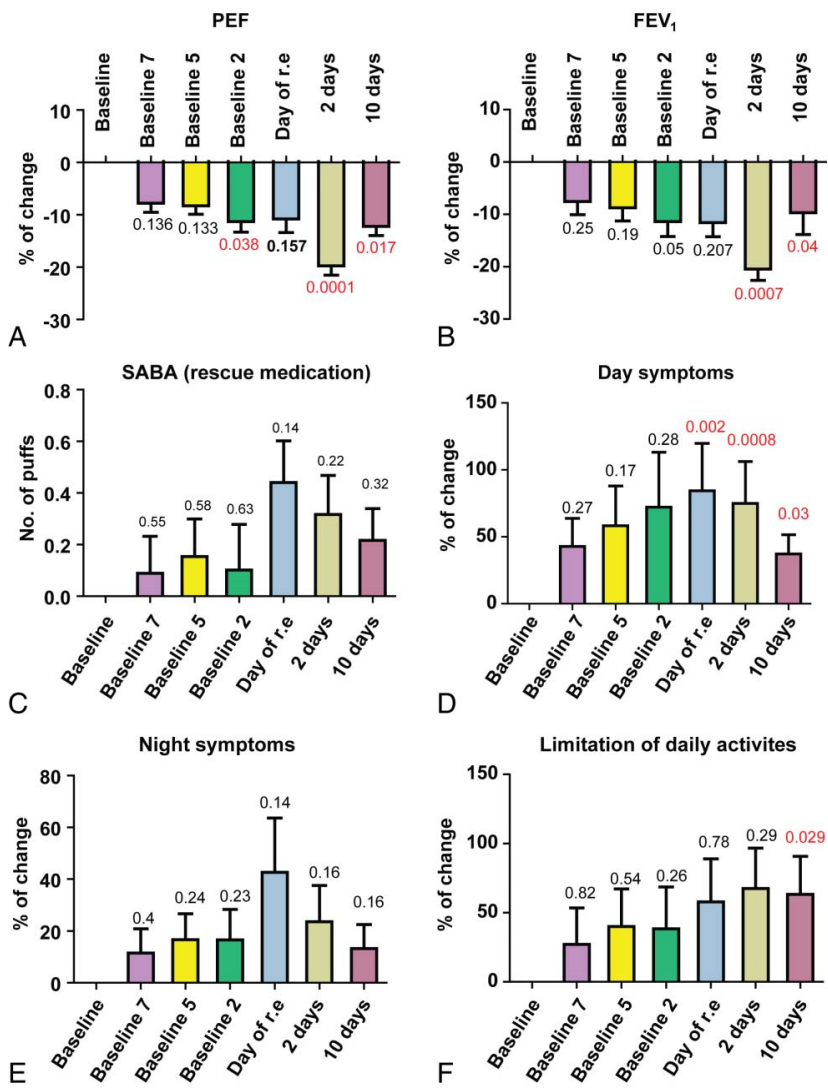

Figure 3 Percentage of change from baseline (=personal best during optimisation phase of the study, calculated as average of the last 10 days of the treatment optimisation period) for the different studied variables during exacerbations in patients with severe exacerbations.

(A) Peak expiratory flow (PEF), (B) forced expiratory volume in $1 \mathrm{~s}$ $\left(\mathrm{FEV}_{1}\right),(\mathrm{C})$ rescue medication (change in number of puffs), (D) day symptoms, (E) night symptoms and (F) limitation of daily activities; error bars represent SEM, Mann-Whitney test. Day or r.e.: the day of reported exacerbations which represents values measured during an unscheduled visit to the clinic due to symptoms of exacerbations ( 1 day); 2 days: the average of the values from the two worst consecutive days during exacerbation; 10 days: the average of 10 days during exacerbations; $2,5,7$ days baseline: the average of $-2,-5$ or -7 days before an exacerbation. SABA, short-acting $\beta$ agonist. This figure is only reproduced in colour in the online version.

\section{Sensitivity and specificity of evaluated outcome variables to detect a SE}

ROC curves for the percentage decrease in PEF and $\mathrm{FEV}_{1}$ versus baseline values in SEs are presented in figure 4. Testing previously used cut-off points, such as a $30 \%$ or $20 \%$ decrease in $\mathrm{PEF}^{8}$ resulted in a sensitivity of $29 \%$ and $45 \%$ respectively, and a specificity of $92 \%$ and $85 \%$ respectively (table 2 ). A $30 \%$ or $20 \%$ decrease in $\mathrm{FEV}_{1}$ resulted in a sensitivity of $31 \%$ and $49 \%$ respectively, and a specificity of $92 \%$ and $82 \%$ respectively. The sensitivity and specificity of different cut-off points for other tested variables (change in $\mathrm{FEV}_{1}$, increase in rescue drug use, increase in day and night symptoms) for the whole study group are presented in table 2 .

We also assessed whether the combination of two or more variables increased the sensitivity and specificity of a given definition to detect exacerbations. Based on the analyses of single variables (see above), combinations that were most likely to increase the sensitivity and specificity were selected. Thus, the combination of a $20 \%$ decrease in PEF on 2 consecutive days and/or a 20\% increase in day symptoms on 2 consecutive days (combined predictors 

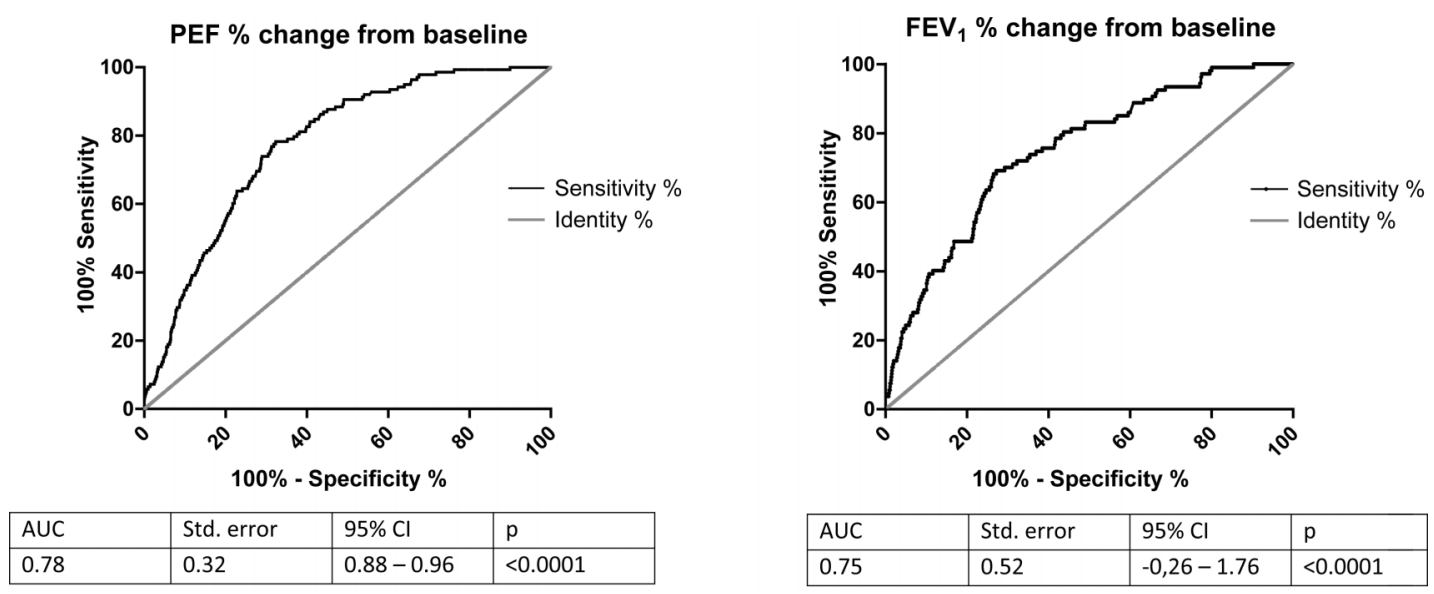

Figure 4 Receiver-operator characteristic curves for change in peak expiratory flow (PEF) and forced expiratory volume in $1 \mathrm{~s}$ (FEV f $_{1}$ as variables to detect physician-diagnosed exacerbations in the whole asthma group. To build receiver-operator characteristics curves, change in a certain variable measured on the first two days of a doctor-diagnosed exacerbation vs baseline was compared to the change in a certain variable measured on all normal days outside of the exacerbation period vs baseline for all studied variables (PEF, FEV 1 , day and night symptoms, and rescue medication use). AUC, area under the curve.

used), and the combination of a $20 \%$ decrease in $\mathrm{FEV}_{1}$ and/or a $20 \%$ increase in day symptoms on 2 consecutive days (combined predictors used) were evaluated (table 3). The highest sensitivity and specificity were found when an exacerbation was defined as a combined 20\% decrease in PEF on 2 consecutive days and a $20 \%$ increase in day symptoms on 2 consecutive days. This definition was able to detect a SE with a sensitivity of $65.0 \%$ and a specificity of $94.9 \%$. Using $\mathrm{FEV}_{1}$ instead of PEF as a lung function variable in combination with day symptoms gave the same specificity but a slightly lower sensitivity (60.4\%).

\section{Baseline subject characteristics associated with the development of exacerbations}

The univariate analysis of factors (baseline patient characteristics) associated with the development of SEs, or any

Table 2 Sensitivity and specificity of selected cut-off points for the parameters evaluated to detect severe exacerbations

\begin{tabular}{|c|c|c|}
\hline \multirow[b]{2}{*}{ Parameter } & \multicolumn{2}{|l|}{ Severe exacerbations } \\
\hline & Sensitivity \% (95\% Cl) & Specificity \% (95\% Cl) \\
\hline \multicolumn{3}{|c|}{ Decrease in PEF (\% change vs baseline) } \\
\hline 30 & $29(21.6$ to 37.0$)$ & $92(91.5$ to 92.0$)$ \\
\hline 20 & 45 (36.0 to 53.6$)$ & 85 (84.8 to 85.7$)$ \\
\hline 15 & 54 (45.6 to 62.8$)$ & $80(79.6$ to 81.0$)$ \\
\hline \multicolumn{3}{|c|}{ Decrease in $\mathrm{FEV}_{1}$ (\% change vs baseline) } \\
\hline 30 & $31(22.3$ to 40.5$)$ & $92(91.0$ to 92.0$)$ \\
\hline 20 & 49 (38.8 to 82.0$)$ & $82(81.3$ to 82.7$)$ \\
\hline 15 & 63 (53.7 to 72.6$)$ & 75 (74.0 to 75.7$)$ \\
\hline \multicolumn{3}{|c|}{ Increase in SABA use (number of puffs) } \\
\hline 2 & $4(2.2$ to 5.7$)$ & 97 (96.6 to 97.3 ) \\
\hline 1 & $4(2.4$ to 5.9$)$ & 96 (95.5 to 96.3 ) \\
\hline \multicolumn{3}{|c|}{ Increase in day symptoms (\% change vs baseline) } \\
\hline 30 & $44(34.5$ to 53.0$)$ & 84.6 (84.6 to 86.0$)$ \\
\hline 20 & 46 (37.0 to 55.6$)$ & 84.9 (83.4 to 84.7$)$ \\
\hline \multicolumn{3}{|c|}{ Increase in night symptoms (\% change vs baseline) } \\
\hline 30 & $34(25.8$ to 44.0$)$ & $85(84.2$ to 85.5$)$ \\
\hline 20 & 41 (31.6 to 50.4$)$ & $82(81.3$ to 82.7$)$ \\
\hline
\end{tabular}

exacerbations, in the whole asthma group and SA group are presented in online repository table E5. When adjusted for possible confounding factors (age, smoking, atopy and gender) in multivariate logistic regression models (table 4), Juniper ACQ score $>1.36$, sputum eosinophils $\geq 3 \%$, BMI $>25$ and SGRQ score $>34.6$ were associated with an increased risk of SEs. Factors associated with an increased risk of developing any exacerbation in our asthma cohort were a history of smoking, SGRQ score $>34.6$, Juniper ACQ score $>1.36$, atopy and reversibility $\geq 12 \%$. In the SA group a history of smoking, female gender, age $<65$ years and Juniper ACQ score $>2.14$ were associated with an increased risk of developing an exacerbation. Having a BMI $>30$ was associated with a decreased risk for the development of exacerbations in SA.

\section{DISCUSSION}

Guidelines recognise the prevention of asthma exacerbations as a major goal of asthma treatment. ${ }^{1}$ The ATS/ERS Task Force on asthma control and exacerbations highlighted the absence of studies aimed at validating objective criteria for the capturing of

Table 3 Sensitivity and specificity of combined parameters to detect severe exacerbations

\begin{tabular}{|c|c|c|}
\hline \multirow[b]{2}{*}{ Definition of exacerbation } & \multicolumn{2}{|c|}{$\begin{array}{l}\text { Severe exacerbations in the } \\
\text { whole asthma cohort }\end{array}$} \\
\hline & $\begin{array}{l}\text { Sensitivity } \\
(\%)\end{array}$ & $\begin{array}{l}\text { Specificity } \\
(\%)\end{array}$ \\
\hline $\begin{array}{l}20 \% \text { decrease in PEF on } 2 \text { consecutive days } \\
\text { and } 20 \% \text { increase in day symptoms on } 2 \\
\text { consecutive days }\end{array}$ & 13.3 & 99.5 \\
\hline $\begin{array}{l}20 \% \text { decrease in PEF on } 2 \text { consecutive days or } \\
20 \% \text { increase in day symptoms on } 2 \\
\text { consecutive days }\end{array}$ & 65.0 & 94.9 \\
\hline $\begin{array}{l}20 \% \text { decrease in } \mathrm{FEV}_{1} \text { on } 2 \text { consecutive days } \\
\text { and } 20 \% \text { increase in day symptoms on } 2 \\
\text { consecutive days }\end{array}$ & 13.2 & 99.3 \\
\hline $\begin{array}{l}20 \% \text { decrease in } \mathrm{FEV}_{1} \text { on } 2 \text { consecutive days or } \\
20 \% \text { increase in day symptoms on } 2 \\
\text { consecutive days }\end{array}$ & 60.4 & 94.8 \\
\hline
\end{tabular}

Bold used to underline the most significant parts of the definition. $\mathrm{FEV}_{1}$, forced expiratory volume in $1 \mathrm{~s}$; PEF, peak expiratory flow. 
Table 4 Adjusted ORs in multivariate logistic regression models for factors associated with the development of severe exacerbations and any exacerbations in the whole asthma cohort and severe asthma group

\begin{tabular}{|c|c|c|c|c|}
\hline Factor & OR & $95 \% \mathrm{Cl}$ & p Value & Comments \\
\hline \multicolumn{5}{|l|}{ Severe exacerbations } \\
\hline Juniper ACQ >1.36 (median) & 3.61 & 1.7 to 7.65 & 0.001 & Adjusted for age, gender, smoking and atopy \\
\hline Sputum eosinophils $\geq 3 \%$ & 3.27 & 1.13 to 9.42 & 0.028 & As above \\
\hline BMI $>25$ & 2.9 & 1.3 to 6.5 & 0.01 & As above \\
\hline SGRQ >34.6 (median) & 2.22 & 1.03 to 4.8 & 0.042 & As above \\
\hline \multicolumn{5}{|l|}{ Whole study group } \\
\hline Smoking history & 3.69 & 1.66 to 8.21 & 0.001 & Adjusted for age, gender and atopy \\
\hline SGRQ >34.6 (median) & 3.09 & 1.41 to 6.76 & 0.005 & Adjusted for age, gender, smoking and atopy \\
\hline Juniper ACQ >1.36 (median) & 3.07 & 1.49 to 6.29 & 0.002 & Adjusted for age, gender, smoking and atopy \\
\hline Atopy & 2.06 & 1.01 to 4.19 & 0.047 & Adjusted for age, gender and smoking \\
\hline Reversibility $\geq 12 \%$ & 2.04 & 1.0 to 4.14 & 0.049 & Adjusted for age, gender, smoking and atopy \\
\hline Sputum eosinophils $\geq 3 \%$ & 2.39 & 0.89 to 6.37 & 0.081 & As above \\
\hline $\mathrm{FEV}_{1} \leq 80 \%$ & 1.93 & 0.95 to 3.93 & 0.071 & As above \\
\hline \multicolumn{5}{|l|}{ Severe asthma } \\
\hline Smoking history & 9.14 & 2.47 to 33.87 & 0.001 & Adjusted for age, gender and atopy \\
\hline Female gender & 4.47 & 1.55 to 12.84 & 0.005 & Adjusted for age, smoking and atopy \\
\hline Age $<65$ years & 4.28 & 1.03 to 17.82 & 0.046 & Adjusted for gender, smoking and atopy \\
\hline Juniper ACQ >2.14 (median) & 3.58 & 1.14 to 11.29 & 0.029 & Adjusted for age, gender, smoking and atopy \\
\hline $\mathrm{BMI}>30$ & 0.28 & 0.09 to 0.87 & 0.028 & As above \\
\hline SGRQ >44.1 (median) & 2.465 & 0.85 to 7.17 & 0.098 & As above \\
\hline
\end{tabular}

There were no statistically significant factors found in the mild to moderate asthma group.

$A C Q$, Asthma Control Questionnaire; BMI, body mass index; FEV 1 , forced expiratory volume in $1 \mathrm{~s}$; SGRQ, St George's Respiratory Questionnaire.

exacerbations. ${ }^{8}$ In this study, we analysed electronic diary data collected over a 1-year period from 169 patients with asthma. We assessed how changes in the recorded outcomes related to 122 physician-diagnosed exacerbations determined during the study period, independently of the information provided by the patients in the diaries. We found that when evaluating individual outcomes changes in lung function (PEF and $\mathrm{FEV}_{1}$ ) gave the highest sensitivity and specificity as a proxy for exacerbations, whereas day and night symptoms, and in particular use of rescue medication, were less suitable as indicators of an exacerbation (table 2). Moreover, the combined criteria of a $20 \%$ decrease in PEF on 2 consecutive days or a $20 \%$ increase in day symptoms on 2 consecutive days, further increased sensitivity and specificity to close to $65 \%$ and $95 \%$, respectively (table 3 ).

The strength of the study lies in the careful patient selection, prospective design and 1-year follow-up period. Strict diagnostic criteria were applied to recruit a group of patients in whom comorbidities and poor compliance with treatment were unlikely to confound the study results. In addition, all patients underwent a 4-week treatment optimisation period with high doses of inhaled corticosteroids and long-acting $\beta$ agonists before the follow-up year, making it highly likely that each subject's personal best lung function and asthma control were determined. Moreover, all information was collected using electronic diaries and the data were related to the time points for independently physician-diagnosed exacerbations. This approach was recommended by the ATS/ERS Task Force, ${ }^{8}$ and significantly increases the quality of the database compared with paper diaries and other strategies in which data are usually collected retrospectively, and therefore more likely to contain errors. 8 13-15

Previous definitions of exacerbations have used a wide panel of unvalidated or partly validated criteria. ${ }^{8}$ Most commonly, a decline in PEF of $20-30 \%$ has been applied. Usually at least 2 consecutive days with a lower PEF are required; however, in some studies a single day with a low PEF measurement was accepted. ${ }^{16}$ Furthermore, changes from baseline PEF, rather than from on-treatment PEF values, have been used. In our study, the average PEF value obtained after 2 weeks of treatment optimisation were used as a baseline for further analyses. Gelb et $a l^{17}$ evaluated the use of lung function parameters for identifying patients with asthma at higher risk of exacerbation and found that using an $\mathrm{FEV}_{1}$ value of $76 \%$ predicted as a cut-off value resulted in a sensitivity of $91 \%$ and a specificity of $50 \%$. However, we found that a change in PEF provides a somewhat higher sensitivity and specificity than $\mathrm{FEV}_{1}$, and moreover, is easier to measure outside the hospital setting. Using a $20 \%$ decrease in PEF compared with the baseline personal best gave a higher sensitivity than using $30 \%$ as a cut-off point. This agrees with the findings of Tattersfield et al, ${ }^{18}$ in which a fall of at least $20 \%$ in PEF was seen in $69 \%$ of all exacerbations, whereas a fall in PEF of at least $30 \%$ only occurred in $45 \%$. Similarly, Chan-Yeung et $a l^{19}$ found that a $30 \%$ decrease in PEF was too stringent a criterion for defining an acute exacerbation.

In our study, all electronic diary card data started to change a week before the recorded exacerbation (figure 3), supporting our strategy of recording personal best values to increase sensitivity. In the trial by Tattersfield et $a l^{18}$ a similar pattern of changes in PEF, symptoms and $\beta$-agonist use prior to exacerbations was found, suggesting that none of these measures provides an earlier warning of an approaching exacerbation. In our study, an increase in day symptoms gave a higher sensitivity and specificity than night symptoms, thus this variable was incorporated into the combined definition. To our surprise, rescue medication (SABA) use gave the lowest sensitivity and specificity of all factors studied. This result is presumably due to changes in clinical practice in Europe during the last decade with widespread use of LABAs. This has caused a significant decrease in the use of SABAs as a rescue drug in patients with SA. The results of our study suggest that an increase in SABA use, due to 
its low sensitivity, should not be recommended as a variable for detecting exacerbations in asthma. However, it needs to be acknowledged that self-reported inhaler use poorly reflects actual use as determined by electronic monitoring with a tendency to under-report, especially regarding overusage.

The use of mathematical correlations and distribution properties of electronic lung function data have previously been used to define the probability of exacerbations. ${ }^{20} 21$ Thamrin et $a l^{22}$ proved that lung function history quantified by fluctuation analysis provides additional information and may help characterise the current state of asthma control. Taking into account the results of our study, we propose that monitoring of electronic lung function data together with other variables such as symptoms, may further increase the prediction of exacerbations in real time. In the future, such strategies incorporated into specific software for smartphones could be integrated into an asthma self-management plan to improve the quality of healthcare and optimise the level of asthma control. ${ }^{23}$ The current finding of high sensitivity for the combination of $20 \%$ decrease in PEF or $20 \%$ increase of symptoms supports use of such an algorithm to define a SE and thus to serve as a signal for the patient to contact the healthcare provider. Perhaps the addition of baseline patient profiles to such strategies may further enhance the effectiveness of monitoring by providing individualised levels of alert. For example, in our SA cohort, the multivariate statistics indicated that women with low baseline asthma control were particularly exacerbation prone.

One of the limitations of our study may be the possibility that not all exacerbations were captured and reported during the 1-year follow-up. Thus, some events might have been treated at home without contact with the supervising clinical centre, as some patients may have occasionally followed their own prestudy personalised asthma action plans. To address this issue we performed a post hoc analysis of the data from the electronic diaries. With the application of our 20\%PEF+20\%Sx (symptoms) exacerbation definition, we found 155 additional events in the SA group and 6 in the MA group that could be regarded as possible non-reported exacerbations. It is remarkable, however, that all those possible missed events were found in the very same patients who had already been identified as having exacerbations in the SA and MA cohorts. No extra events were identified in subjects who did not have physician-verified exacerbations, and the allocation to the analysed groups was the same. Thus, we believe that this has not had any impact on our analysis of the specificity and sensitivity of different indicators, but is more likely to have resulted in an underestimation of the number of exacerbations per patient. Taken together, the post hoc analysis supports the validity of the ' $20 \% \mathrm{PEF}+20 \% \mathrm{Sx}$ ' algorithm and also supports previous indications that patients with exacerbations represent one specific phenotype. ${ }^{24}$

In conclusion, the results of our study indicate that monitoring of PEF and symptoms of asthma gives the highest sensitivity and specificity for use as a proxy of SEs in asthma. Increased use of rescue medications (SABA) was of little value for capturing exacerbations, presumably due to recent changes in therapeutic routines.

\footnotetext{
Author affiliations

${ }_{1}^{1}$ Centre for Allergy Research, Karolinska Institutet, Stockholm, Sweden

${ }^{2}$ Department of Respiratory Medicine, University of Amsterdam, Amsterdam, The Netherlands

${ }^{3}$ Department of Medicine, Jagiellonian University, Krakow, Poland

${ }^{4}$ Department of Clinical and Experimental Medicine, University of Ferrara, Ferrara, Italy

${ }^{5}$ Department of Respiratory Diseases, University of Marseille, Marseille, France
}

${ }^{6} 7^{\text {th }}$ Respiratory Department and Asthma Centre, University of Athens, Athens, Greece

${ }^{7}$ Italian National Research Council, Palermo, Italy

${ }^{8}$ Infection, Inflammation and Immunology Division, University of Southampton, Southampton, UK

${ }^{9}$ Airway Disease Infection Section, National Heart and Lung Institute, Imperial College, London, UK

${ }^{10}$ Department of Respiratory Medicine, Ghent University Hospital, Ghent, Belgium

${ }^{11} \mathrm{KLB}$ Health Research, Luebeck, Germany

${ }^{12}$ Department of Thoracic Medicine, Medical School, University of Crete, Heraklion, Greece

Acknowledgements We would like to thank colleagues at the Karolinska Institutet library for their help.

Collaborators BIOAIR — Longitudinal Assessment of Clinical Course and Biomarkers in Severe Chronic Airway Disease: Amsterdam: Els Weersink, Athens: Nikos Papadopoulos, Erasmia Oikonomidou, Eleftherios Zervas, Ferrara: Marco Contoli, Ghent: Romain A. Pauwels, ${ }^{\dagger}$ Guy Brusselle, Isabelle de Rudder, Vanessa Schelfhout, Hamburg/Grosshansdorf: Kai Richter, Daisy Gerding, Helgo Magnussen, Heraklion: Nikos M. Siafakas, Katerina Samara, Maria Plataki, Eva Papadopouli, Krakow: Andrzej Szczeklik, ${ }^{\dagger}$ Bozena Ziolkowska-Graca, Aleksander Kania, Agnieszka Gawlewicz-Mroczka, Mariusz Duplaga, Ewa Figiel, Leiden: Klaus F. Rabe, Pieter S. Hiemstra, Stefanie Gauw, Ilonka van Veen, Leuven: Johan C. Kips, London: Patrick Mallia, Deborah A Campbell, Douglas S Robinson, Modena: Leo M. Fabbri, Micaela Romagnoli, Montpellier: Isabelle Vachier, Catherine Devautour, Lahouari Meziane, Palermo: A. Maurizio Vignola, ${ }^{\dagger}$ Elisabetta Pace, Mirella Profita, Southampton: Stephen T. Holgate, Susan J. Wilson, Lorraine Hewitt, John Holoway, Stockholm: Katarina Damm, Ingrid Delin, Marianne Eduards, Alexandra Ek, Tommy Ekström, Agneta Gülich, Lovisa E. Johansson, Östen Karlsson, Maria Kumlin, Ingrid Martling, and Maria Skedinger.

tDeceased.

Contributors Primary data aggregation and statistical analyses were done by MK, SH and SED, and then discussed and interpreted with the other coauthors at face-to-face meetings. MK wrote the first draft of the manuscript. Revisions were made by MK together with SED and in an interactive process including all coauthors. All authors participated in the study design, conduction of the trial, collection and interpretation of data, and critical review of the draft versions of the report.

Funding The BIOAIR study was supported by The Fifth and Sixth Framework Programmes of the European Union, contract numbers QLG1-CT-2000-01185 (BIOAIR) and FOOD-CT-2004-506378 (GA 2 LEN), and several national funding bodies (Sweden: Heart-Lung Foundation, Asthma and Allergy Foundation and the Stockholm County Council; Greece: unrestricted competitive research grant 'The Herakleitos project 2002', from the Hellenic Ministry of Education; UK: Patrick Mallia was supported by unrestricted grant from GSK). Maciej Kupczyk is a scholarship fellow of the Wenner-Gren Foundations and is supported by the Bernard Osher Initiative for Research on Severe Asthma at the Karolinska Institutet. The BIOAIR study received unconditional support from AstraZeneca Sweden, Vitalograph Inc, Aerocrine $A B$ and Amedon $\mathrm{GmbH}$.

\section{Competing interests None.}

Ethics approval The local ethics committees and drug regulatory authorities in 12 participating centres.

Provenance and peer review Not commissioned; externally peer reviewed.

\section{REFERENCES}

1 Global Initiative for Asthma. Global strategy for asthma management and prevention. 2008. http://www.ginasthma.com (accessed 25 Mar 2013).

2 Holgate ST, Polosa R. The mechanisms, diagnosis, and management of severe asthma in adults. Lancet 2006;368:780-93.

3 Bousquet J, Mantzouranis E, Cruz AA, et al. Uniform definition of asthma severity, control, and exacerbations: document presented for the World Health Organization Consultation on Severe Asthma. J Allergy Clin Immunol 2010;126:926-38.

4 Bel EH, Sousa A, Fleming L, et al. Diagnosis and definition of severe refractory asthma: an international consensus statement from the Innovative Medicine Initiative (IMI). Thorax 2011;66:910-17.

5 The ENFUMOSA Study Group. The ENFUMOSA cross-sectional European multicentre study of the clinical phenotype of chronic severe asthma. Eur Respir J 2003:22:470-7.

6 Moore WC, Bleecker ER, Curran-Everett D, et al. Characterization of severe asthma phenotype by the National Heart, Lung and Blood Institute's Severe Asthma Research Program. J Allergy Clin Immunol 2007;119:405-13.

7 Hedlin G, Bush A, Carlsen K Lodrup, et al. Problematic severe asthma in children, not one problem but many: a GA²LEN initiative. Eur Respir J 2010;36:196-201. 
8 Reddel HK, Taylor DR, Bateman ED, et al. An official American Thoracic Society/ European Respiratory Society statement: asthma control and exacerbations. Am J Respir Crit Care Med 2009;180:59-99.

9 Kupczyk M, Haque S, Middelveld R, et al. Exacerbations in severe asthma-1 year follow up in the BIOAIR study. Am J Respir Crit Care Med 2011;183:A2247.

10 Juniper EF, O'Byrne PM, Guyatt GH, et al. Development and validation of a questionnaire to measure asthma control. Eur Respir J 1999;14:902-7.

11 Jones PW, Quirk FH, Baveystock CM, et al. A self-complete measure of health status for chronic airflow limitation. The St George's Respiratory Questionnaire. Am Rev Respir Dis 1992;145:1321-7.

12 Kupczyk M, Middelveld R, Holgate $\mathrm{S}$, et al. The BIOAIR study-European trial on severe asthma. Eur Respir J 2012; in press.

13 Lane S, Molina J, Plusa T. An international observational prospective study to determine cost of asthma exacerbations (COAX). Respir Med 2006;100:434-50.

14 Cote J, Cartier A, Malo JL, et al. Compliance with peak expiratory flow monitoring in home management of asthma. Chest 1998;113:968-72.

15 van der Meer V, Rikkers-Mutsaerts ERVM, Sterk PJ, et al. Compliance and reliability of electronic PEF monitoring in adolescents with asthma. Thorax 2006:61:457-8.

16 Reddel HK, Jenkins CR, Marks GB, et al. Optimal asthma control, starting with high doses of inhaled budesonide. Eur Respir J 2000;16:226-35.
17 Gelb AF, Taylor CF, Shinar CM, et al. Role of spirometry and exhaled nitric oxide to predict exacerbations in treated asthmatics. Chest 2006;129:1492-99.

18 Tattersfield AE, Postma DS, Barnes PJ, et al. Exacerbations of asthma. A descriptive study of 425 severe exacerbations. Am J Respir Crit Care Med 1999;160:594-9.

19 Chan-Yeung M, Chang JH, Manfreda J, et al. Changes in peak flow, symptom score, and the use of medications during acute exacerbations of asthma. Am J Respir Crit Care Med 1996;154:889-93.

20 Frey U, Brodbeck T, Majumdar A, et al. Risk of severe asthma episodes predicted from fluctuation analysis of airway function. Nature 2005;438:667-70.

21 Thamrin C, Zindel J, Nydegger $\mathrm{R}$, et al. Predicting future risk of asthma exacerbations using individual conditional probabilities. J Allergy Clin Immunol 2011;127:1494-502.

22 Thamrin C, Nydegger R, Stern G, et al. Associations between fluctuations in lung function and asthma control in two populations with differing asthma severity. Thorax 2011:66:1036-42.

23 Kupczyk M, Dahlen SE. Will recording of lung function fluctuation open the door to internet-guided treatment of asthma? Thorax 2011;66:1019-20.

24 ten Brinke A, Sterk PJ, Masclee AA, et al. Risk factors of frequent exacerbations in difficult-to-treat asthma. Eur Respir J 2005;26:812-18. 